\title{
End-to-end performance of DF multihop hybrid RF/FSO system using MPPM coding and MIMO technique under dependent GG turbulence channels
}

\author{
Hechmi Saidi, Noureddine Hamdi \\ ElManar Farhat Hached University Campus, Tunisia
}

\begin{tabular}{|c|c|}
\hline Article Info & ABSTRACT \\
\hline Article history: & The paper presents a BER analysis for hybrid Radio Frequency/ multihop \\
\hline Received Nov 20, 2019 & Free Space Optics, known as RF/ FSO, communication system benefiting \\
\hline Recerved ivor zo, zory & from Multiple Input Multiple Output (MIMO) configuration but impaired by \\
\hline Revised Jan 26, 2020 & Gamma-Gamma, known as GG, turbulence. Authors deploy Multiple Pulse \\
\hline Accepted Feb 11, 2020 & $\begin{array}{l}\text { Position Modulation, known as M-PPM, like a signaling technique } \\
\text { Furthermore, the exact as well as approximated amounts of Symbol-Erro }\end{array}$ \\
\hline Keywords: & $\begin{array}{l}\text { Rates, known as SERs, are both judged in this work. A closed form formula } \\
\text { related to the whole Probability Density Function, called PDF, for our }\end{array}$ \\
\hline FSO & $\begin{array}{l}\text { designed system is derived. Such interesting approach provides to us better } \\
\text { quality of service by combating atmospheric turbulence. }\end{array}$ \\
\hline GG channel & \\
\hline Hybrid & \\
\hline MIMO & \\
\hline $\mathrm{RF}$ & $\begin{array}{r}\text { Copyright }\left({ }^{\circ} 2020 \text { Institute of Advanced Engineering and Science. }\right. \\
\text { All rights reserved. }\end{array}$ \\
\hline \multicolumn{2}{|l|}{ Corresponding Author: } \\
\hline \multicolumn{2}{|c|}{$\begin{array}{l}\text { Hechmi Saidi, } \\
\text { ElManar Farhat Hached University Campus, } \\
\text { Tunis Belvedere 1002, Tunisia. } \\
\text { Email: hechmi.saidi@rns.tn }\end{array}$} \\
\hline
\end{tabular}

\section{INTRODUCTION}

The FSO communication affords cost-effective means for achieving huge capacity and is used in various telecommunication applications [1-2]. One of the most crucial occurrences that influence FSO system's performance is scintillation [3-4]. This system involves also light signal transmission over atmospheric turbulence channels [5]. GG distributions, eventually, are valid for a larger variety of such turbulence strength from weak to strong [6].

To combat atmospheric turbulence and enhance the transmitted signal quality, the MIMO technique's virtues have motivated the researchers to integrate several transmit and receive sensors in FSO communication scheme [7]. MPPM favours the power efficiency over the bandwidth [8]. With the aim of describing the transmitted signal appropriately at the receiver, many statistical and mathematical channels models are investigated in [9-15]. Moreover, the outage probability's closed-form expression for a MIMO FSO system using binary PPM has been illustrated in [16]. For independent channels, the GG derived signals' result combined summation can be estimated by the $\alpha-\mu$ distribution [17]. DF relaying is considered in [18-20] whereas Quantize and Forward (QF) one is used in [21].

We are interested to combine such techniques to improve the QoS of FSO communication system by dealing with a new configuration which is RF/MIMO FSO multihop network over GG turbulence correlated channels and using MPPM modulation scheme. Exact and approximated values of SERs are developed for our system with a closed form formula for the PDF expression. This paper is arranged as follow: The scheme model is introduced in subsection 2.1. We also present in this section its statistical model. In subsection 2.2, we express the end to end BER. Section 3 discusses the results with a conclusion in Section 4. 


\section{RESEARCH METHOD}

\subsection{System model}

Hybrid DF multihop RF/ MIMO FSO network with $\mathrm{N}$ optical sources and $\mathrm{N}$ optical detectors as shown in Figure 1.

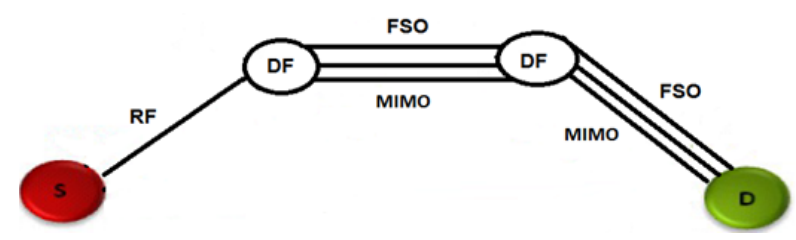

Figure 1. Hybrid DF multihop RF/ MIMO FSO network with $\mathrm{N}$ optical sources and $\mathrm{N}$ optical detectors

GG channel distribution overview:

The GG distribution's marginal PDF is introduced like in [6]:

$$
\begin{gathered}
g\left(K_{s}\right)=\frac{2(x y)^{\frac{x+y}{2}}}{\Omega \Gamma(x) \Gamma(y)}\left(\frac{K_{s}}{\Omega}\right)^{\frac{x+y}{2}-1} B_{x-y}\left(2 \sqrt{\frac{x y K_{s}}{\Omega}}\right), \\
\text { where } K_{s} \geq 0,
\end{gathered}
$$

and knowing that $\tau$ (.) represents gamma function, $\mathrm{Bc}($.$) describes cth order case of modified Bessel function$ related to second kind, Ks illustrates average received information photon count for each signal slot and $\Omega=$ $\mathrm{E}^{\left\{k_{s}\right\}}$ is Ks's expected mean. We denote $\mathrm{x}$ and $\mathrm{y}$ as GG distribution's format parameters, associated to refractive as well as diffractive turbulence impacts with large-scale as well as small-scale eddies effective number. $x_{s c}^{2}:=\operatorname{var}\left\{k_{s}\right\} / E\left\{k_{s}^{2}\right\}$ denotes scintillation index function of $(\mathrm{x}, \mathrm{y})$ :

$$
\chi_{S C}^{2}=\frac{1}{x}+\frac{1}{y}+\frac{1}{x y}
$$

Concerning weak towards moderate turbulence, $X_{s c}^{2}$ belongs to $[0,0.75]$, whereas $X_{s c}^{2}>0.75$ case of strong turbulence [5].

MIMO-FSO model

Our M-PPM symbol period is composed of Q intervals (time slots). Besides, an optical power seems solely transmitted during $\mathrm{w}$ times slots, where $\mathrm{w}$ belongs to $\{1,2, \ldots, \mathrm{Q} / 2\}$. We transmit each MPPM frame through all $\mathrm{N}$ transmitters. Using equal gain combining, the average received photon count for every signal slot is described as

$$
K_{S_{o n}}=\sum_{m=1}^{N} \sum_{n=1}^{N} K_{S_{m n}}+K_{b}
$$

where Ksmn represents average received signal photon count concerning mth source as well as nth receiver, $\mathrm{Kb}$ denotes average received photon count for every slot because of background noise. Each Q summation of N2 GG random variables has a PDF as:

$$
Z^{j}=\sum_{m=1}^{N} \sum_{n=1}^{N} Y_{m n}^{j}, j \in\{1,2, \ldots, Q\}
$$

where $Y_{m n}^{j}$ denotes depicted photon count during jth slot having $j \in\{1,2, \ldots . ., Q\}$ having both $\mathrm{n}$ and $m \in\{1,2, \ldots . ., \mathrm{N}\}$. MIMO configuration 
Having a spacing between detectors often lower than correlation distance, $(\alpha-\mu)$ distribution is invalid for $\mathrm{Z}$ of (4). Thus, the PDF closed-form expression case of equal gain combining dependent MIMO channels in Figure 1 could be mathematically described [22]:

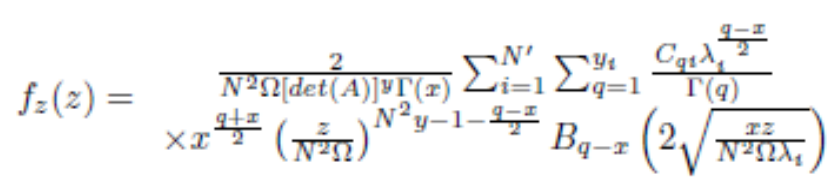

$\left\{\lambda_{i}\right\}_{i=1}^{N^{2}}$ represent eigenvalues for A=DC, where D mentioned $\mathrm{N}^{2} * \mathrm{~N}^{2}$ diagonal matrix including small-scale form for the factor $\mathrm{y}$, while $\mathrm{C}$ describes $\mathrm{N}^{2} * \mathrm{~N}^{2}$ positive specific association matrix including $\sqrt{\rho_{i j}}$ correlation coefficients. N0 denotes the number case of matrix A's different eigenvalues $\lambda_{i}$. Besides, $y_{i}=\mu_{A}\left(\lambda_{i}\right) y$, where $\mu_{A}\left(\lambda_{i}\right)$ represents eigenvalue's algebraic multiplicity. Thus, the coefficient cqi could be provided as:

$$
c_{q i}=\sum_{k_{k_{1}+\cdot \hat{i}^{*}+k_{N^{\prime}}=y_{i}-q} \frac{\left(\begin{array}{c}
y_{i}-q \\
k_{1}, \cdot \hat{i}_{i}, k_{N^{\prime}}
\end{array}\right)}{\left(y_{i}-q\right) !}}^{\prod_{j=1, j \neq i}^{N^{\prime}}\left[(-1)^{k_{j}}\left(y_{j}\right) k_{j}\left(d_{j}-d_{i}\right)^{-\left(y_{j}+k_{j}\right)}\right]}
$$

$d_{j}=\frac{z}{N^{2} \Omega \lambda_{f}}\left(\begin{array}{l}y_{i}-q \\ k_{1, \ldots, i}, k_{N^{\prime}}\end{array}\right)=\frac{y_{i}-q}{k_{1, \ldots, i}, k_{N^{\prime}} !}$

means that $k_{i}$ is deleted from the sequence, and $\left(y_{j}\right)_{k_{j}=\frac{r\left(y_{j}+k_{j}\right) !}{r\left(y_{j}\right)}}$ represents Pochhammer symbol RF link

The received pulse $y(t)$ could be defined like a function of transmitted one $x(t)$ and channel response $h(t)$ as follows [23]:

$$
y(t)=x(t) * h(t)=\int_{-\infty}^{\infty} x(\tau) h(t-\tau) d \tau
$$

Whereas the AWGN noise can be modeled as in [23]:

$$
f(x, \mu, \sigma)=\frac{1}{\sqrt{2 \pi} \sigma} e^{-\left[\frac{(x-\mu)^{2}}{2 \sigma^{2}}\right]}
$$

With x: random variable and ${ }^{\mu}$ its meanwhile

$\sigma$ : normalized fluctuation.

\subsection{Performance analysis} MIMO-FSO portion

SER:

Having a received vector described like

$$
Z=\left(Z^{1}, Z^{2}, \ldots . ., Z^{Q}\right)
$$

where $\mathrm{Zj}$, whose $j \in\{1,2, \ldots ., Q\}$, denotes a summation over jth slot. In addition to such Q slots, we have w ON ones for carrying data while $Q-\omega$ OFF ones for no information. Let 


$$
P_{M I N}=p_{1}\left(z_{\min }\right)^{m}\left(1-P_{1}\left(z_{\min }\right)\right)^{w-m}
$$

Knowing that zmin represents lowest photon amount per symbol signal time slots. Whereas, p1(.) represents probability of photon count for signal time slots. P1(.) denotes its cumulative statistical distribution. If a number of one or else more among OFF slots has count $>=$ to that case of ON slots, we have a transmission error. The SER case of MPPM coding in absence of turbulent atmosphere path is introduced as in [24]:

$$
\begin{gathered}
\text { SER }=\sum_{z_{\min }=0}^{\infty} \sum_{l=1}^{Q-w} \sum_{m=1}^{w}\left(\begin{array}{c}
w \\
m
\end{array}\right)\left(\begin{array}{c}
Q-\mid w \\
l
\end{array}\right) \\
\times P_{M I N} P_{0}\left(z_{\min }-1\right)^{Q-w-l}\left[\left(1-P_{0}\left(z_{\min }\right)\right)^{l}+p_{0}\left(z_{\min }\right)^{l}\right. \\
\left.\left(1-\frac{1}{\left(\begin{array}{c}
l+m \\
m
\end{array}\right)}\right)\right]
\end{gathered}
$$

Where $\mathrm{p} 0($.$) represents probability of photon count for non-signal slots. Whereas, \mathrm{P} 0($.$) represents its$ cumulative statistical distribution. Adopting Poisson distribution, the above probabilities are described as:

$$
\begin{gathered}
p_{0}(k)=\frac{K_{b}^{k}}{k !} e^{-K_{b}}, p_{1}(k)=\frac{\left(z+K_{b}\right)^{k}}{k !} e^{-\left(z+K_{b}\right)} \\
P_{0}(k)=\sum_{j=0}^{k} \frac{K_{b}^{j}}{j !} e^{-K_{b}}, P_{1}(k)=\sum_{j=0}^{k} \frac{\left(z+K_{b}\right)^{j}}{j !} e^{-\left(z+K_{b}\right)} \ldots
\end{gathered}
$$

Having $\mathrm{k}$ a positive integer, $\mathrm{Z}$ an average received data photon amount by $\mathrm{ON}$ time slot. Regular SER might be determined as a result of averaging (9) considering $\mathrm{z}$ value. Assuming that solely PMIN in (9) depends on our channel statistical distribution, we can obtain average SER from replacing PMIN by its average $\mathrm{P} 2(\mathrm{zmin})$ in (9):

$$
P_{2}\left(z_{\min }\right)=\int_{0}^{\infty} p_{1}\left(z_{\min }\right)^{m}\left(1-P_{1}\left(z_{\min }\right)\right)^{w-m} f_{Z}(z) d z
$$

which after algebraic manipulations can be expressed as:

$$
\begin{gathered}
P_{2}\left(z_{\min }\right)=\sum_{j=(w-m)\left(z_{\min }+1\right)}^{\infty} \sum_{B=0}^{j+m z_{\min }}\left(\begin{array}{c}
j+m z_{\min } \\
B
\end{array}\right) \\
r(j) \times \frac{e^{-w k_{b} k_{b}^{j+m z_{m i n}-B}}}{z_{\min } !^{m}} \int_{0}^{\infty} z^{B} e^{-w z} f_{Z}(z) d z
\end{gathered}
$$

Whose $\mathrm{r}(\mathrm{j})$, such as $j \geq(\omega-m)\left(Z_{\min }+1\right)$, is described like

$$
\begin{aligned}
& r(j)=\sum_{\left(S_{1}, S_{2}, \ldots, S_{w-m}\right) \in \chi(j)} \frac{1}{S_{1} ! S_{2} ! \ldots S_{w-m} !} \\
& \text { over the set of vectors } \chi(j) \text {, where } \\
& \chi(j)=\left(S_{1}, S_{2}, \ldots, S_{w-m}\right) \in N^{w-m}: \sum_{i=1}^{w-m} S_{i}= \\
& j \text { and } \forall \ell \in\{1,2, \ldots, w-m\}, z_{\min }+1 \leq S_{\ell} \leq \\
& j-(w-m-1)\left(z_{\min }+1\right)
\end{aligned}
$$

Exact SER:

By replacing (5) and (6) into (12), the average SER of MIMO-FSO dependent channels could be rewritten from [25]: 


$$
\begin{aligned}
& P_{2}\left(z_{\min }\right)=\sum_{j=(w-m)\left(z_{m i n}+1\right)}^{+\infty} \sum_{B=0}^{j+m z_{m i n}} \sum_{i=1}^{N^{\prime}} \sum_{q=1}^{y_{i}} \\
& \sum_{k_{1}+\cdot \hat{\mathrm{i}}+k_{N^{\prime}}=y_{1}-q}\left(\begin{array}{c}
y_{i}-q \\
k_{1}+. . \hat{i}_{\hat{i}} \cdot k_{N^{\prime}}
\end{array}\right) \times\left(\begin{array}{c}
j+m z_{\min } \\
B
\end{array}\right) \\
& \exp \left(\frac{x}{2 N^{2} \Omega \lambda_{1} \omega}-\omega K_{b}\right) \omega^{-\left(B+N^{2} y+\frac{q+x}{2}-\sum_{j=1}^{N^{\prime}} y_{j}-\frac{1}{2}\right)}
\end{aligned}
$$

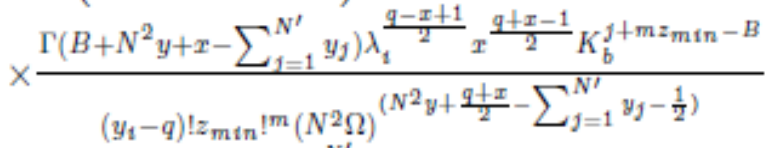

$$
\begin{aligned}
& \times \frac{r(j) \Gamma\left(B+N^{2} y+q-\sum_{j=1}^{N^{\prime}} y_{j}\right)}{[\operatorname{det}(A)]^{y} \Gamma(q) \Gamma(x)} \\
& \times \prod_{j=1, j \neq i}^{N^{\prime}}\left[(-1)^{k_{j}}\left(y_{j}\right)_{k_{j}}\left(\frac{1}{\lambda_{j}}-\frac{1}{\lambda_{i}}\right)^{-\left(y_{j}+k_{j}\right)}\right] \\
& \times W-\left(B+N^{2} y+\frac{q+x}{2}-\sum_{j=1}^{N^{\prime}} y_{j}-\frac{1}{2}\right)\left(\frac{q-x}{2}\right)\left(\frac{x}{N^{2} \Omega \lambda_{i} W}\right)
\end{aligned}
$$

having $\mathrm{W}(.,).($.$) as Whittaker function$

Approximate SER:

Through Gauss-Laguerre method for quadrature law [5].We can rewrite (11) like:

$$
\begin{aligned}
P_{2}\left(z_{\min }\right)= & \left.\frac{e^{\left(-w k_{b}\right)}}{z_{\min ! !}} \int_{0}^{\infty} e^{(}-w z\right)\left(z+k_{b}\right)^{m z_{\min }} \times\left[e^{\left(z+k_{b}\right)}\right. \\
& \left.-\sum_{B=0}^{j+m z_{m i n}} \frac{\left(z+k_{b}\right)^{j}}{j !}\right]^{w-m} f_{z}(z) d z
\end{aligned}
$$

This integration might be estimated due to Gauss-Laguerre approach:

$$
\begin{aligned}
P_{2}\left(z_{\min }\right) \approx \sum_{i=1}^{c} \wedge_{i} \frac{\left.e^{(}-w k_{b}\right)}{z_{\min } ! m}\left(\mathrm{~V}_{i}+k_{b}\right)^{m z_{m i n}} & \\
& \times\left[e^{\left(\vee_{i}+k_{b}\right)}\right. \\
-\sum_{j=0}^{z_{\min }} & \left.\frac{\left(\vee_{i}+k_{b}\right)^{j}}{j !}\right]^{w-m} f_{z}\left(\vee_{i}\right) d z
\end{aligned}
$$

where Vi represents $\mathrm{i}$-th basis for the Laguerre polynomial $\mathrm{Lc}(\mathrm{x})$ where $\mathrm{c}>1 .{ }^{\Lambda_{i}}$ denotes the corresponding weighting coefficient.

RF portion

BER:

Our bit error function could be defined [23]:

$$
P(e)=Q\left(\left[2\left(\frac{N_{0}}{E_{S}}\right)+4 B \Delta\left(\frac{N_{0}}{E_{S}}\right)^{2}\right]^{-\frac{1}{2}}\right)
$$

with E S: received energy per symbol

$\mathrm{Q}(\mathrm{x})$ : null mean Gaussian distribution function

N0: AWGN's single-sided power spectral density

$B$ : signals bandwidth

$\mathrm{T}$ : integration time.

The End to End BER

We have, in our model, 3 independent symmetric portions with an error probability Pi for the ith hop $(i \in\{1,3\})$.We obtain an $\operatorname{E2E~SEP~}\left(P_{e}^{t}\right)$ as:

$$
P_{e}^{t}=1-\left(1-P_{1}\right)\left(1-P_{2}\right)\left(1-P_{3}\right)=1-(1-P(e))\left(1-P_{2}\left(z_{\min }\right)\right)^{2}
$$




\section{RESULTS AND DISCUSSION}

In this section, we assumed that $\mathrm{M}=\mathrm{N}=2$, Laguerre polynomial degree $\mathrm{c}=100$ and having 8PPM modulation scheme under weak and strong GG atmospheric turbulence (respectively $\sigma_{R}=\{0.25,0.75\}$ ). In Figure 2, we remark that average SER(ASER) diminishes when average SNR (ASNR) augments. Besides, when we have greater correlation coefficient, we have lower system quality. When $\rho \rightarrow 1$ (high correlation), having channels affected by identical fading and then receiving identical signals, there is no difference between MIMO and SISO configurations in term of QoS. Nevertheless, when $\rho \rightarrow 0$ (low correlation), all channels are nearly independent and thus the system takes complete virtue of MIMO diversity leading to better QoS. Figure 3 showed in fact that when the number of hops increased which means that the distance between resource and destination was growing; the ASER seemed to be little worse but didn't affect so much the whole system performance. Thanks to multihop configuration, we could send data for far distances.

For example, for single hop for moderate turbulence, we had ASER $=10-11$ when the ASNR $=100$ while for three-hop we had 10-11< SER < 10-10 which means that SER3hops \& SER1hop under same turbulence and for the same SNR. Indeed, we gained $2 \mathrm{Km}$ (the distance between two nodes $=1 \mathrm{Km}$ ) more having almost the same quality of service i.e. the same data rate. In addition, we found in Figure 4 that the all RF links seemed the most reliable one with less ABER than hybrid one which is better than all FSO one. To note here that FSO looks more important to repair last mile breakdown at the absence of fiber-optic communications like complement for RF networks.

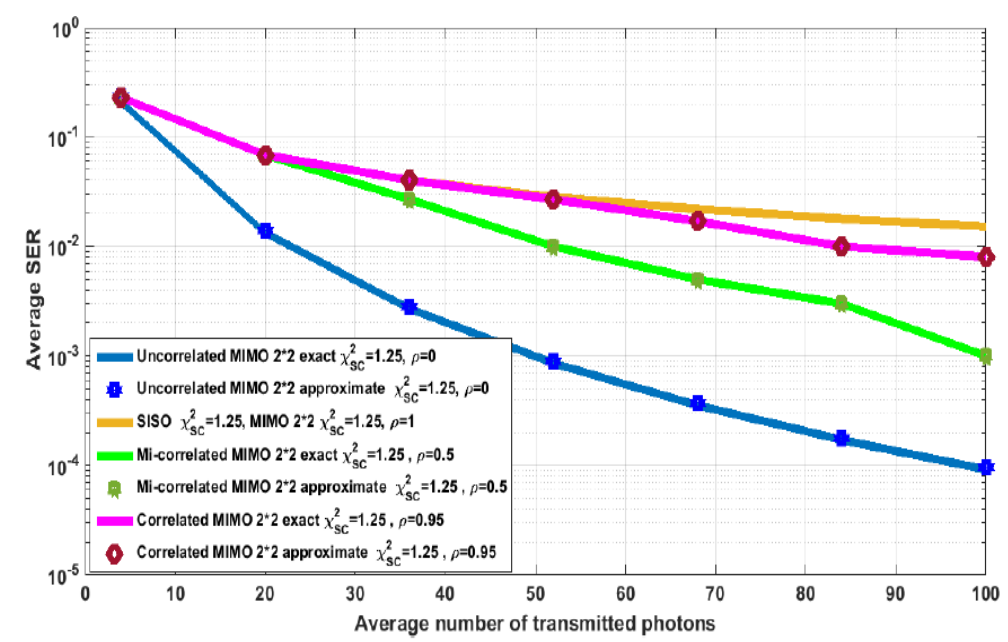

Figure 2. ASER versus ASNR for different correlated MIMO channels parameters under strong turbulence

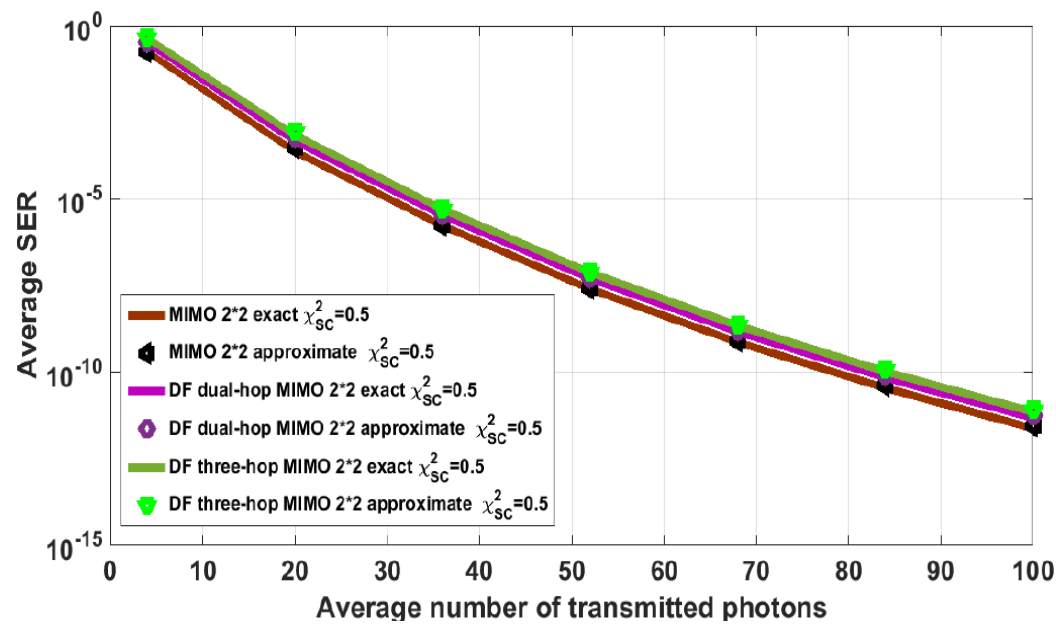

Figure 3. Multihop MIMO-FSO system QoS under moderate turbulence where $\rho=0.1$ 


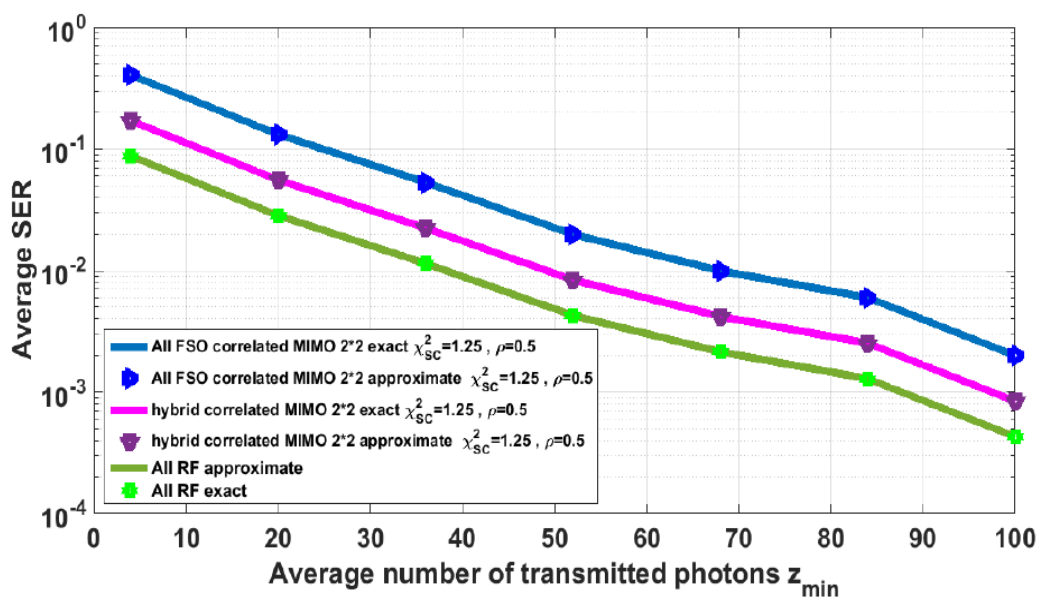

Figure 4. Comparison between multihop DF relaying only MIMO FSO or only RF or hybrid RF/MIMO FSO

\section{CONCLUSION}

We have formulated a closed-form expression for BER of Hybrid RF/ SO system. Thanks to the use of hybrid configuration, MIMO and MMPM signaling, the effect of many impairments had been combatted. The numerical results helped to corroborate such result. In fact, the great problem for our multihop FSO system is the complexity of serial AF multihop mathematics analysis. It is now required to go deeper into the analysis and into the study of the boost of its performance. Moreover, the considering error pointing effect would be another outlook for this work

\section{REFERENCES}

[1] Sajid. S. M., Tomaž J., Igor J., Erich L., Otto K. "Reed Solomon coded ppm for terrestrial fso links". 2007 International Conference on Electrical Engineering. Lahore. pp. 1-5. 2007.

[2] Ahmed B., Md R. I., Mohamed H., Suriza A. "Availability Prediction Methods for Terrestrial Free-Space-Optical Link Under Tropical Climate". Indonesian Journal of Electrical Engineering and Computer Science, vol. 10, no. 1, pp. 224-229. 2018

[3] Scott B, Eric K, John S, Heinz W. "Understanding the performance of free-space optics". Journal of optical Networking vol. 2, no. 6, pp.178-200, 2003.

[4] Xiaoming Z, Joseph M. K. "Communications techniques and coding for atmospheric turbulence channels". Journal of Optical and Fiber Communications Reports, vol. 4, no. 6, pp. 363-405. 2007.

[5] Kamran K. "Performance of APD-based, PPM free-space optical communication systems in atmospheric turbulence". IEEE transactions on communications, vol. 53, no. 9, pp. 1455-1461, 2005.

[6] Ammar A, Larry C. A, Ronald L. P. "Mathematical model for the irradiance probability density function of a laser beam propagating through turbulent media". Optical Engineering, vol. 40, pp. 1554-1562, 2001.

[7] Manav R. B. "A one bit feedback based beamforming scheme for fso miso system over gamma-gamma fading". IEEE Transactions on Communications, vol. 63, no. 4, pp. 1306-1318, 2015.

[8] Hiroaki S, Kiyoshi N. "MPPM: A method for improving the bandutilization efficiency in optical PPM". Journal of Lightwave Technology, vol. 7, no. 3, pp. 465-472, 1989.

[9] Stephen G. W., Maite B. P., Qialing C, Michael B. "Optical repetition MIMO transmission with multipulse PPM". IEEE journal on Selected Areas in Communications, vol. 23, no. 9, pp. 1901-1910, 2005.

[10] Seyed M. N., Murat U., Mohsen K. "Ber performance of free space optical transmission with spatial diversity". IEEE Transactions on wireless communications, vol. 6, no. 8, 2007.

[11] Hossein K., Murat U., "Performance analysis of mimo free-space optical communication systems with selection combining". IEEE 21st Signal Processing, Communication and Applications Conference (SIU). Haspolat, pp. 1-4. 2013.

[12] Neda C., Stephen G. W, Maite B. P. "Performance bounds for free-space mimo systems with apd receivers in atmospheric turbulence". IEEE Journal on Selected Areas in Communications, vol. 26, no. 3, pp. 3-12, 2008.

[13] Shuai J., Guowei Y., Yizhen W., Meihua B, Yang L., Xuefang Z., Miao H, Qiliang L., "Performance analysis of space-diversity free space optical links over exponentiated weibull channels". IEEE Photonics Technology Letters, vol. 27, no. 21, pp. 2250-2252, 2015.

[14] Prabhmandeep K., Vk. J., Subrat K. "Performance analysis of free space optical links using multi-input multioutput and aperture averaging in presence of turbulence and various weather conditions". IET Communications, vol. 9(8): 1104-1109. 2015.

[15] Chadi A. R., "Performance analysis of fso communications with diversity methods: Add more relays or more apertures". IEEE Journal on Selected Areas in Communications, vol. 33, no. 9, pp. 1890-1902, 2015. 
[16] Hossein K., Zohreh M., Murat U., Zabih G. Outage performance of mimo free-space optcal systems in gamma-gamma fading channels. Proceedings of the 2013 18th European Conference on Network and Optical Communications \& 2013 8th Conference on Optical Cabling and Infrastructure (NOC-OC\&I). Graz, pp. 275-280. 2013

[17] Kostas P. P. A simple, accurate approximation to the sum of gamma gamma variates and applications in mimo freespace optical systems. IEEE Photonics Technology Letters, vol. 23, pp. 839-941, 2011.

[18] Milica I. P., Aleksandra M. C., Goran T. D., George K. K. Partial relay selection with outdated channel state estimation in mixed RF/FSO systems. IEEE Journal on selected areas in Communications, vol. 33, no. 13, pp. 2860-2867, 2015.

[19] Muhammad Z. F. K. F., Elsheikh M. A. E. AWGN and Rayleigh Fading Behavior of the Wireless Decode-andForward Relay Channel with Arbitrary Time and Power Allocation. Indonesian Journal of Electrical Engineering and Computer Science, vol. 10, no. 1, pp. 248-257, 2018.

[20] Tariq M. A., Elsheikh M. A. E. Positioning of a Wireless Relay Node for Useful Cooperative Communication. Indonesian Journal of Electrical Engineering and Computer Science, vol. 10, no. 3, 959-965, 2018.

[21] Kuldeep. K., Deva K. B. Quantize and encode relaying through fso and hybrid fso/rf links. IEEE Transactions on Vehicular Technology, vol. 64, no. 6, pp. 2361-2374, 2015.

[22] José M. G. B., Antonio J. N., José F. P., Miguel C. V. Antonio P. N., Spatially correlated gamma-gamma scintillation in atmospheric optical channels. Optics Express, vol. 22, no. 18, pp. 21820-21833, 2014.

[23] Hechmi S., Noureddine H. On the effect of misalignment fading in DF dual-hop hybrid FSO/RF system using MPPM coding method under GG Channel turbulence model. $201814^{\text {th }}$ International Wireless communication \& Mobile computing (IWCMC). Limassol, pp. 566-570, 2015.

[24] E. Morra, H. S. Khallaf, H. M. Shalaby, and Z. Kawasaki, "Performance analysis of both shot-and thermal-noise limited multipulse ppm receivers in gamma-gamma atmospheric channels" Journal of Lightwave Technology, vol. 31, no. 19, pp. 3142-3150, 2013.

[25] Israil. S. G., Jossif M. R. Table of integrals, series, and products. Seventh English edition. Florida, pp. 1200, 2007. 